\section{OCULAR DISEASE AND HIV IN AFRICA}

The scourge of HIV continues nearly uncontrolled in Africa. Available data suggest that ocular disease caused by AIDS is different in Africa. Cytomegalovirus (CMV) retinitis is much less common whereas herpes zoster ophthalmicus and conjunctival squamous cell tumours are more prevalent. Beare and coworkers studied 307 patients with HIV infection; $36 \%$ had tuberculosis and $17 \%$ myobacteraemia. Of those with tuberculosis $94 \%$ had HIV. Surprisingly, choroidal granulomas were found in only four patients, all of whom had clinically obvious AIDS. Seventeen per cent of patients with AIDS had microangiopathy yet only one had signs of active CMV infection. The reason for the rarity of CMV retinitis in Africa is generally considered to be due to mortality of people with AIDS early in the disease before or shortly after the onset of profound immunosuppression. Alternatively it has been suggested that differences in race, HIV subtype, and comorbidity may influence the occurrence of CMV retinitis.

See p 1076

\section{LATANOPROST INDUCED IRIS COLOUR CHANGE}

Increased pigmentation of the iris is a well known side effect of both naturally occurring prostaglandins and prostaglandin analogues, such as isopropyl unoprostone and latanoprost. The reported incidence of increased pigmentation of the iris after long term therapy with latanoprost has varied from 10 $25 \%$ of patients. Teus and coworkers examined the anterior segment of both eyes of 43 patients with glaucoma after the unilateral use of latanoprost. Independent masked observers analysed the slides of both eyes of the study group and compared the iris pigmentation of both eyes using a stereo viewer. Surprisingly, nearly $70 \%$ of patients had a definite acquired asymmetric pigmentary change associated with the eye that had been treated with latanoprost. Approximately $50 \%$ of these patients had increased superficial iris pigmentation

www. bjophthalmol.com with a granular appearance and another $50 \%$ had an increased stromal pigmentation. This study indicates that the incidence of iris pigmentation induced by latanoprost therapy is considerably higher than previously reported. Recent studies suggest that latanoprost increases the number of melanocytes synthesising melanin and melanin content of these cells. Pigment changes associated with latanoprost may be related to activation of melanogenesis in some superficial melanocytes, which increased their melanin content.

See p 1085

\section{OLDER DRIVERS: ARE THEY SAFE TO DRIVE?}

The general perception of the younger population is that in the age group of 80 years and over unsafe driving is the norm. This may of course be an unsubstantiated prejudice of younger drivers. Nevertheless, in this study Keeffe and coworkers analysed driving related questions including a population based study that tried to determine the prevalence and incidence of eye disease. Participants aged 44 years and over were interviewed and subsequently underwent a comprehensive ophthalmic examination. The findings were of interest. Some people with impaired vision either stopped driving voluntarily or when advised to do so. However, the authors found a small portion $(2.6 \%)$ of people in their study with impaired vision who were current drivers. Older drivers generally drove shorter distances and limited their driving to avoid demanding or adverse conditions. There was no greater likelihood of self reported driving accidents from drivers with detectable impaired vision compared to those with good vision. Many older drivers with impaired vision had already limited their driving to avoid adverse conditions and some had stopped driving altogether. This study suggests that visually acuity of less than 6/12 may not be the critical level of visual loss related to road accidents and some other presumably considerably lower visual acuity level might be the appropriate criterion used to determine eligibility to obtain a driver's licence. This is one more piece of evidence which documents that the visual standards for obtaining a driver's licence lack scientific verification with regard to preventing traffic accidents.

See $p 1118$

\section{WHAT DO WE REALLY KNOW ABOUT AMBLYOPIA?}

One would have thought that after the Nobel prize winning studies of Hubel and Wiesel our understanding of the neurophysiology and treatment of amblyopia was complete. Nothing could be further from the truth as several studies have recently demonstrated. Horton has demonstrated in human visual cortex studies that layer 4C of the visual cortex is probably unaffected in strabismic and anisometropic amblyopia. Layer 4C is probably only important for the much less common cases of formed deprivation amblyopia. Then in 1997 Stewart-Brown challenged the ophthalmic community to prove that there was value in preschool vision screening to detect amblyopia and that amblyopia could even be successfully treated. Recent studies in the United Kingdom and the United States done under carefully controlled conditions have now conclusively demonstrated that anisometropic and strabismic amblyopia can be treated effectively during the sensitive period by either occlusion or pharmacological penalisation of the fixing eye. Now Ohlsson and coworkers present data about the stability and long term and visual outcome in amblyopia treatment. In their study 44 children with unilateral amblyopia caused by strabismus or anisometropia were evaluated. They evaluated the stability of visual acuity following the discontinuation of occlusion therapy. Seventeen per cent of patients had deteriorated visual acuity, $50 \%$ were stable yet 33\% actually gained in visual acuity. Interestingly enough there was also an increase in the visual acuity of the non-amblyopic eye in $54 \%$ of patients. These findings are in sharp contrast with findings of Scott and others who have suggested that the so called slippage rate of amblyopes following the cessation of patching therapy is considerably higher than this. Reasons for the differences in these studies is not clear although the relatively short term follow up in the study reported by Ohlsson and coworkers may account for some of it. It is clear that amblyopia remains, at least in part, an enigma that demands further careful clinical study. See $p 1148$ 